\title{
THE VALUATION DIFFERENCE RANK OF A QUASI-ORDERED DIFFERENCE FIELD
}

\author{
SALMA KUHLMANN, MICKAËL MATUSINSKI, AND FRANÇOISE POINT
}

\begin{abstract}
There are several equivalent characterizations of the valuation rank of an ordered or valued field. In this paper, we extend the theory to the case of an ordered or valued difference field (that is, ordered or valued field endowed with a compatible field automorphism). We introduce the notion of difference rank. To treat simultaneously the cases of ordered and valued fields, we consider quasi-ordered fields. We characterize the difference rank as the quotient modulo the equivalence relation naturally induced by the automorphism (which encodes its growth rate). In analogy to the theory of convex valuations, we prove that any linearly ordered set can be realized as the difference rank of a maximally valued quasi-ordered difference field. As an application, we show that for every regular uncountable cardinal $\kappa$ such that $\kappa=\kappa^{<\kappa}$, there are $2^{\kappa}$ pairwise non-isomorphic quasi-ordered difference fields of cardinality $\kappa$, but all isomorphic as quasi-ordered fields.
\end{abstract}

\section{INTRODUCTION}

The theory of convex valuations and coarsenings of valuations is a special chapter in classical valuation theory. It is a basic tool in algebraic and real algebraic geometry. Surveys can be found in [20, 21] and 23. This special chapter is in turn closely related to ordered algebraic structures, see [7]. In particular, an important isomorphism invariant of an ordered or valued field is its rank as a valued field, which has several equivalent characterizations: via the ideals of the valuation ring, the value group, or the residue field, see [27].

This can be extended to ordered and valued fields with extra structure, giving a characterization completely analogous to the above, but taking into account the corresponding induced structure on the ideals, value group, or residue field. For example, in [14, Chapter 3] the notion of the exponential rank of an ordered exponential field is introduced and analysed in light of the above classical tools. The exponential rank measures the growth rate of the given exponential function, and is thus closely related to asymptotic analysis in the sense of G. H. Hardy [10].

In this paper, we push this analogy to the case of an ordered or valued difference field. We work with quasi-ordered fields, see [6]. In Section 2 we review classical notions and results on ordered or valued fields. We thereby present a uniform approach via quasi-orders, treating simultaneously the cases of ordered and valued fields. Theorem 2.2 gives a characterization of the rank of a quasi-ordered field in terms of coarsenings of its natural valuation. Descending down to the value group

1991 Mathematics Subject Classification. Primary 03C60, 06A05, 12J15: Secondary 12L12, $26 \mathrm{~A} 12$.

Supported by a Research in Paris grant from Institut Henri Poincaré, Konstanz University, Bordeaux 1 University and the Fonds de la Recherche Scientifique FNRS-FRS. 
of the quasi-ordered field, and yet further down to the value set $\Gamma$ of the value group, the rank and principal rank are finally characterized by the chain $\Gamma$, see Theorems 2.7 and 2.12. In Section 3 we start by a key remark regarding equivalence relations defined by monotone maps on chains. We describe in Theorem 3.4 the rank of a quasi-ordered field via the equivalence relations induced by addition and multiplication on the field. This approach allows us to develop in Section 4 the notion of difference compatible valuations and introduce the difference rank. We characterize in Theorem 4.2 the difference rank, in analogy to Theorem 2.2, [26, Lemma 1] is a special case of our Corollary 4.8 on weak isometries. Corollary 4.9 describes the set of fixed points of an automorphism $\sigma$ in terms of its difference rank, whereas Corollary 4.11 examines the special case of $\omega$-increasing or $\omega$-contracting automorphisms. In the last Section 5 we describe the principal difference rank, see Theorem 5.3 and its Corollaries 5.5 5.4 and 5.6. In Theorem 5.8 we construct large families of quasi-ordered difference fields with distinct difference ranks.

Some closing comments are in place. The theory of well-quasi orders [16 is currently a highly developed part of combinatorics with surprising applications in logic, mathematics and computer science. Quasi-ordered algebraic structures are interesting in their own right, and we will continue our investigations of these fascinating objects. Quasi-orders [2] appear in the literature also as preorders, see e.g. [7, p.1]. However we will not use this terminology, in order to avoid confusion with the notion of preorders appearing in real algebraic geometry (e.g. in [15). The theory of quasi-ordered abelian groups is closely related to that of C-groups [12] and has already found interesting applications in $[22$ to the study of the asymptotic couple associated to a valued differential field. Throughout the paper, Hahn groups and Hahn fields play a fundamental role. The group of automorphisms of Hahn structures have been extensively studied, see [3], 8], 11] and [26. In future work, we will analyse the behaviour of the difference rank as function defined on these automorphism groups.

\section{THE RANK OF A QUASI-ORDERED FIELD}

A quasi-order (q.o.) on a set $S$ is a binary relation $\preceq$ which is reflexive and transitive. Throughout this paper, we will deal only with total quasi-order, i.e. either $a \preceq b$ or $b \preceq a$, for any $a, b \in S$. We will omit henceforth 'total'. Note that an order is a q.o which is in addition anti-symmetric. In the latter case, we say that $S$ is an ordered set or a chain. The induced equivalence relation is defined by $a \asymp b$ if and only if ( $a \preceq b$ and $b \preceq a$ ). We shall write $a \prec b$ if $a \preceq b$ but $b \asymp a$ fails. Note that $\preceq$ induces canonically a total order on $S / \asymp$. Conversely if $\asymp$ is an equivalence relation on a set $S$ such that $S / \asymp$ is a total order, then $\asymp$ induces canonically a q.o. on $S$. A subset $E$ of $S$ is $\preceq$-convex if for all $a, b, c$ in $S$, if $a \preceq c \preceq b$ and $a, b \in E$, then $c \in E$. We shall write convex instead of $\preceq$-convex if the context is clear.

A quasi-ordered field $(K, \preceq)$ is a field $K$ endowed with a quasi-order $\preceq$ which satisfies the following compatibility conditions, for any $a, b, c \in K$.

qo1: If $a \asymp 0$, then $a=0$.

qo2: If $0 \preceq c$ and $a \preceq b$, then $a c \preceq b c$.

qo3: If $a \preceq b$ and $b \Varangle c$, then $a+c \preceq b+c$. 
From qo2 one deduces that if $a \preceq b$ and $0 \preceq c \preceq d$, then $a c \preceq b c \preceq b d$, so $a c \preceq b d$.

Given a valuation $w$ on $K$ we denote the valuation ring by $K_{w}$, its group of units $K_{w}^{\times}$by $\mathcal{U}_{w}$, its valuation ideal (i.e. its unique maximal ideal) by $I_{w}$, its value group by $w\left(K^{\times}\right)$and residue field $K_{w} / I_{w}$ by $K w$.

An ordered field $(K, \leq)$ is a q.o. field. The valuation on a valued field $(K, w)$ induces a quasi-order: $a \preceq_{w} b$ if and only if $w(b) \leq w(a)$, i.e. if and only if $a b^{-1} \in K_{w}$. S. Fakhruddin [6] showed that if $\preceq$ is a q.o. on a field $K$, then $\preceq$ is either an order or there is a (unique up to equivalence of valuations) valuation $v$ on $K$ such that $\preceq=\preceq_{v}$. The dichotomy is achieved by considering the equivalence class $E_{1}$ of 1 with respect to $\asymp$. In the order case, $E_{1}=\{1\}$ and $\asymp$ is just equality. The quasi-order is said to be a proper quasi-order (p.q.o.) if $E_{1} \neq\{1\}$. In this case, $E_{1} \neq\{1\}$ is a non-trivial subgroup of $K^{\times}$and $K^{\times} / E_{1}$ is an ordered abelian group. Then $\mathcal{U}_{v}$ is just $E_{1}$ and $v\left(K^{\times}\right)$is $K^{\times} / E_{1}$. In the p.q.o case $a \succeq 0$ for all $a \in K$.

Given two valuations $v$ and $w$ on $K$, recall that $w$ is said to be a coarsening of $v(w$ is coarser than $v$ ) or that $v$ a refinement of $w(v$ is finer than $w)$ if $K_{v} \subseteq K_{w}$. In case the inclusion of the valuation rings is strict, we add the predicate strict in the terminology coarser and finer. Note that $w$ is coarser than $v$ if and only if $a \preceq_{v} b$ implies $a \preceq_{w} b$. If $\sim_{1}$ and $\sim_{2}$ are two equivalence relations defined on the same set, then $\sim_{1}$ is said to be coarser than $\sim_{2}$ (or $\sim_{2}$ finer than $\sim_{1}$ ) if $\sim_{2}$-equivalence implies $\sim_{1}$-equivalence.

Let us now fix a q.o. $\preceq$ on $K$. A valuation $w$ on $K$ is called convex with respect to $\preceq$ if its valuation ring $K_{w}$ is convex. It is called compatible with $\preceq$ (or $\preceq$ is compatible with $w$ or $w$ and $\preceq$ are compatible) if for all $a, b \in K$ :

$$
0 \preceq b \preceq a \quad \Longrightarrow \quad w(a) \leq w(b) \text {. }
$$

Equivalently, $w$ is compatible with $\preceq$ if and only if for all $a, b \in K$ :

$$
0 \preceq b \preceq a \quad \Longrightarrow \quad b \preceq w a .
$$

Remark 2.1.

(i) If $\preceq$ is an order, then this is the usual notion of compatibility for orders and valuations, see e.g. [19], [20], [23], or [24].

(ii) If $\preceq=\preceq_{v}$ is a p.q.o. then $w$ compatible with $\preceq_{v}$ just means that for all $a, b \in K, v(a) \leq v(b) \Longrightarrow w(a) \leq w(b)$. This in turn just means that $K_{v} \subseteq K_{w}$ or $w$ is a coarsening of $v$, equivalently $\asymp_{w}$ is coarser than $\asymp_{v}$.

The following gives the characterization of valuations compatible with a quasi-order. Theorem 2.2 is in complete analogy to the characterization of valuations compatible with an order. So for $\preceq$ an order, we omit the proof and refer the reader to [19, Proposition 5.1], or [20, Theorem 2.3 and Proposition 2.9], or [23, Lemma 3.2.1], or [24, Lemma 7.2] or [5, Proposition 2.2.4].

Theorem 2.2. Let $(K, \preceq)$ be a q.o. field and $w$ a valuation on $K$. The following assertions are equivalent:

1) $w$ is compatible with $\preceq$,

2) $w$ is convex,

3) $I_{w}$ is convex,

4) $I_{w} \prec 1$, 
5) the quasi-order $\preceq$ induces canonically via the residue map $a \mapsto$ aw a quasi-order on the residue field $K w$.

Proof. Assume $\preceq=\preceq_{v}$ is a p.q.o. Compatible valuations are clearly convex, this follows from the definitions. Conversely if $w$ is convex and $0=v(1) \leq v(a)$, i.e. $a \preceq 1$, then $a \in K_{w}$ by convexity. So $w$ is a coarsening of $v$. This establishes the equivalence of 1) and 2).

If $w$ is convex, $a \preceq b$ with $b \in I_{w}$, then $0<w(b) \leq w(a)$ by compatibility, so $a \in I_{w}$. Conversely assume $I_{w}$ convex, and let $a \preceq b$ with $b \in K_{w} \backslash I_{w}$. If $a \notin K_{w}$ then $a^{-1} \in I_{w}$. Now $b^{-1} \preceq a^{-1}$, so $b^{-1} \in I_{w}$, a contradiction. This establishes the equivalence of 2) and 3 ).

If $I_{w}$ is convex, then $w$ is a coarsening of $v$, so $I_{w} \subseteq I_{v} \prec 1$. Conversely, assume $I_{w} \prec 1$ and let $a \preceq b$ with $b \in K_{w}$. If $a \notin K_{w}$, then $a^{-1} \in I_{w}$. So $a^{-1} b \in I_{w}$ whence $a^{-1} b \prec 1$. Multiplying by $a$ gives $b \prec a$, a contradiction. This establishes the equivalence of 3 ) and 4 ).

Now let $w$ be a coarsening of $v$. Then $v$ induces canonically a valuation $v / w$ on the residue field $K w$, defined by $v / w(a w):=\infty$ if $a w=0$ and $v / w(a w):=v(a)$ otherwise ([5] p. 44) . The p.q.o. $\preceq_{v / w}$ is precisely the induced well defined quasiorder in 5), i.e. $a w \preceq_{v / w} b w$ if and only if $a \preceq_{v} b$ holds. Conversely, let $\preceq_{v / w}$ be a p.q.o. on $K w$ induced by the residue map. This means that $a w \preceq_{v / w} b w$ if and only if $a \preceq_{v} b$ holds. Then $w$ is a coarsening of $v$ (see [5, p. 45]). This establishes the equivalence of 1) and 5).

Remark 2.3. If $\preceq$ is an order then the induced quasi-order in 5) is also an order, if $\preceq$ is a p.q.o then the induced quasi-order in 5) is also a p.q.o.

Let $(K, \preceq)$ be a q.o. field. We define its natural valuation, denoted by $v$, to be the finest $\preceq$ - convex valuation of $K$. If $(K, \leq)$ is ordered, then the natural valuation is the valuation $v$ whose valuation ring $K_{v}$ is the convex hull of $\mathbb{Q}$ in $K$. In this case, the natural valuation on $K$ satisfies $v(x+y)=\min \{v(x), v(y)\}$ if $\operatorname{sign}(x)=$ $\operatorname{sign}(y)$ and for all $a, b \in K: a \geq b>0 \Longrightarrow v(a) \leq v(b)$. It is characterized by the fact that the induced order on its residue field $K v$ is archimedean, i.e. the only equivalence classes for the archimean equivalence relation (see definition below following Lemma 2.5) are those of 0 and 1. If $w$ is a coarsening of a convex valuation, then $w$ also is convex. Conversely, a convex subring containing 1 is a valuation ring, see [5, Section 2.2.2]. The set $\mathcal{R}$ of all valuation rings $K_{w}$ of convex valuations $w \neq v$ (i. e. all strict corsenings of $v$ ) is totally ordered by inclusion. Its order type is called the rank of the ordered field $K$. For convenience, we will identify it with $\mathcal{R}$. For example, the rank of an archimedean ordered field is empty since its natural valuation is trivial (i.e. its valuation ring is the field itself). The rank of the rational function field $K=\mathbb{R}(t)$ with any order is a singleton: $\mathcal{R}=\{K\}$. Theorem 2.2 is a characterization of the elements of the rank of the ordered field $(K, \leq)$. Note that the rank of $(K, \leq)$ is invariant under isomorphisms of ordered fields.

If $(K, \preceq)$ is p.q.o. then the unique valuation $v$ such that $\preceq={ }_{v}$ is the natural valuation. A compatible valuation $w$ is a coarsening of $v$. We define the rank of the valued field $(K, v)$ to be the (order type of the) totally ordered set $\mathcal{R}$ of all strict corsenings of $v$. Thus, Theorem 2.2 is a characterization of the elements of the rank of $(K, v)$. Note that the rank of $(K, v)$ is invariant under isomorphisms of valued 
fields. As we recalled in the proof of Theorem 2.2, the natural valuation $v$ induces canonically a valuation $v / w$ on the residue field $K w$ and $v$ is the compositum of $w$ and $v / w$ (see [5, pp. 44-45]). The p.q.o. $\preceq_{v / w}$ is precisely the induced quasi-order in Theorem 2.25). If $w=v$, then $v / w$ is trivial. Thus $v$ is characterized by the fact that the induced p.q.o on its residue field $K v$ is trivial, i.e. the only equivalence classes of $\asymp$ are those of 0 and 1 .

Remark 2.4. The maximal ideals $I_{w}$ appearing in Theorem 2.2 4) are prime ideals of the valuation ring $K_{v}$. The strict coarsenings $K_{w}$ of $K_{v}$ are the localizations of $K_{v}$ at the prime ideals $\{0\} \subseteq I \subset I_{v}$, [5. Lemma 2.3.1 p. 43], [27, Theorem 15, p. 40]. Thus the rank is also isomorphic to the totally ordered (by reverse inclusion) set of prime ideals of $K_{v}$ which are strictly contained in the maximal ideal $I_{v}$.

We now want to characterize the rank by going down to the value group. Let $v$ be the natural valuation on the q.o. field $(K, \preceq)$. We set $G=v\left(K^{\times}\right)$. Recall that the set of all convex subgroups $G_{w} \neq\{0\}$ of the value group $G$ is totally ordered by inclusion. Its order type is called the rank of $G$, it is an isomorphism invariant, see [7] or [23. To every convex valuation ring $K_{w}$, we associate a convex subgroup $G_{w}:=\{v(a) \mid a \in K \wedge w(a)=0\}=v\left(\mathcal{U}_{w}\right)$. We call $G_{w}$ the convex subgroup associated to $w$. Note that $G_{v}=\{0\}$. Conversely, given a convex subgroup $G_{w}$ of $v\left(K^{\times}\right)$, we define $w: K \rightarrow v\left(K^{\times}\right) / G_{w}$ by $w(a)=v(a)+G_{w}$. Then $w$ is a convex valuation with $v\left(\mathcal{U}_{w}\right)=G_{w}$ (and $v$ is strictly finer than $w$ if and only if $G_{w} \neq\{0\}$ ). We call $w$ the convex valuation associated to $G_{w}$. We summarize the above discussion in the following lemma, for more details see [5], or [7] or [23].

Lemma 2.5. The correspondence $K_{w} \mapsto G_{w}$ is an order preserving bijection, thus $\mathcal{R}$ is (isomorphic to) the rank of $G$.

We now want to characterize the rank by going further down to the value set of the value group. Recall that on the negative cone $G^{<0}$ of an ordered abelian group $G$, the archimedean equivalence relation $\sim$ is defined by: $a \sim b$ if and only if there is $n \in \mathbb{N}$ such that $a \geq n b$ and $b \geq n a$. Let $v_{G}$ be the map $a \mapsto[a]_{\sim}$, where $[a]_{\sim}$ denotes the equivalence class of $a$. The order on $\Gamma:=G^{<0} / \sim$ is the one induced by the order of $G^{<0}$. We call $v_{G}\left(G^{<0}\right):=\Gamma$ the value set of $G$. By convention we also write $v_{G}(G):=\Gamma \cup\{\infty\}$ extending the archimedean equivalence relation to the positive cone of $G$ by setting $v_{G}(g):=v_{G}(-g)$ and $v_{G}(0)=\infty>\Gamma$. The map $v_{G}$ on $G$ satisfies the ultrametric triangle inequality, and in particular we have: $v_{G}(x+y)=\min \left\{v_{G}(x), v_{G}(y)\right\}$ if $\operatorname{sign}(x)=\operatorname{sign}(y)$. We call $v_{G}$ the natural valuation on $G$.

We now recall the relation between the rank of $G$ and the value set $\Gamma$ of $G$. To $G_{w} \neq\{0\}$ a convex subgroup, we associate $\Gamma_{w}:=v_{G}\left(G_{w}^{<0}\right)$ a non-empty final segment of $\Gamma$. Conversely, if $\Gamma_{w}$ is a non-empty final segment of $\Gamma$, then $G_{w}=\{g \mid$ $\left.g \in G, v_{G}(g) \in \Gamma_{w}\right\} \cup\{0\}$ is a convex subgroup, with $\Gamma_{w}=v_{G}\left(G_{w}\right)$. Let us denote by $\Gamma^{\mathrm{fs}}$ the set of non-empty final segments of $\Gamma$, totally ordered by inclusion. We summarize the above discussion in the following lemma, for more details see [5], or [7] or [23.

Lemma 2.6. The correspondence $G_{w} \mapsto \Gamma_{w}$ is an order preserving bijection, thus the rank of $G$ is (isomorphic to) $\Gamma^{\mathrm{fs}}$.

Combining Lemmas 2.5 and 2.6 we obtain the following result. Note that Theorem 2.7 will also follow, by a different argument, from Theorem 3.4 in the next section. 
Theorem 2.7. The correspondence $K_{w} \mapsto \Gamma_{w}$ is an order preserving bijection, thus $\mathcal{R}$ is (isomorphic to) $\Gamma^{\mathrm{fs}}$.

A final segment which has a least element is a principal final segment. It is of the form $\left\{\gamma^{\prime} \mid \gamma^{\prime} \in \Gamma, \gamma^{\prime} \geq \gamma\right\}$, for some $\gamma \in \Gamma$. Let $\Gamma^{*}$ denote the set $\Gamma$ with its reversed ordering. The proof of the following Lemma is now routine.

Lemma 2.8. The map from $\Gamma$ to $\Gamma^{\mathrm{fs}}$ defined by $\gamma \mapsto\left\{\gamma^{\prime} \mid \gamma^{\prime} \in \Gamma, \gamma^{\prime} \geq \gamma\right\}$ is an order reversing embedding. Its image is the set of principal final segments. Thus $\Gamma^{*}$ is (isomorphic to) the totally ordered set of principal final segments.

For the notions and results in this last paragraph of the section, we refer the reader to 7] or [23] for more details. Recall that a convex subgroup $G_{w}$ of $G$ is called principal generated by $g, g \in G$, if $G_{w}$ is the minimal convex subgroup containing $g$. The principal rank of $G$ is the subset of the rank of $G$ consisting of all principal $G_{w} \neq\{0\}$.

Lemma 2.9. Let $G_{w} \neq\{0\}$ be a convex subgroup. Then $G_{w}$ is principal if and only if $v_{G}\left(G_{w}\right)=\Gamma_{w}$ is a principal final segment.

Lemma 2.10. The map $G_{w} \mapsto \min \Gamma_{w}$ is an order reversing bijection from the principal rank of $G$ onto $\Gamma$. Thus the principal rank of $G$ is (isomorphic to) $\Gamma^{*}$.

We set: $\mathbf{P}_{K}:=K^{\succeq 0} \backslash K_{v}$, where $K^{\succeq 0}:=\{a \in K ; a \succeq 0\}$. A $K_{w} \in \mathcal{R}$ is principal generated by $a$ for $a \in \mathbf{P}_{K}$ if $K_{w}$ is the smallest (convex) subring containing $a$. We observe:

Lemma 2.11. Let $K_{w} \in \mathcal{R}$. Then, $K_{w}$ is principal generated by $a$ if and only if $K_{w}=\left\{b \in K: \exists n \in \mathbb{N}_{0}\right.$ s.t. $\left.b \preceq_{v} a^{n}\right\}$.

Proof. It is enough to verify that $\left\{b \in K: \exists n \in \mathbb{N}_{0} \quad b \preceq_{v} a^{n}\right\}$ is a subring of $K$. Let $b_{1} \preceq_{v} a^{n_{1}}$ and $b_{2} \preceq_{v} a^{n_{2}}$. Then $b_{1} b_{2} \preceq_{v} a^{n_{1}+n_{2}}$ and $b_{1}+b_{2} \preceq_{v} a^{\max \left\{n_{1}, n_{2}\right\}}$. Clearly, this ring contains $K_{v}$ and $a$.

The principal rank of $K$ is the subset $\mathcal{R}^{\text {pr }}$ of $\mathcal{R}$ consisting of all principal $K_{w} \in \mathcal{R}$. Combining the last three lemmas we obtain:

Theorem 2.12. The correspondence $K_{w} \mapsto \Gamma_{w}$ is an order preserving bijection between $\mathcal{R}^{\mathrm{pr}}$ and the principal rank of $G$, thus $\mathcal{R}^{\mathrm{pr}}$ is (isomorphic to) $\Gamma^{*}$.

Note that Theorem 2.12 will also follow, by a different argument, from Theorem 3.4 in the next section.

Remark 2.13. It is straightforward to verify that an order preserving isomorphism $\psi: \Gamma_{1} \rightarrow \Gamma_{2}$ induces an order preserving isomorphism $\psi^{\mathrm{fs}}: \Gamma_{1}^{\mathrm{fs}} \rightarrow \Gamma_{2}^{\mathrm{fs}}([25$, p.19]). Thus $\Gamma$ determines $\Gamma^{\mathrm{fs}}$ up to isomorphism. It follows from Theorems 2.7 and 2.12 that if two q.o. fields have isomorphic principal ranks, then they have isomorphic ranks. In the next section we shall hence focus our attention on the principal rank.

\section{The PRINCIPAL RANK VIA EQUIVALENCE RELATIONS}

We begin by the following key observation: 
Remark 3.1. Let $\varphi$ be a map from a q.o. ordered set $(S, \preceq)$ into itself, and assume that $\varphi$ is q.o. preserving, i. e. $a \preceq a^{\prime}$ implies $\varphi(a) \preceq \varphi\left(a^{\prime}\right)$, for all $a, a^{\prime} \in S$. Assume that $\varphi$ has an orientation or is oriented, i. e. $\varphi(a) \succeq a$ for all $a \in S$ ( $\varphi$ is a right shift) or $\varphi(a) \preceq a$ for all $a \in S$ ( $\varphi$ is a left shift $)$. We set $\varphi^{0}(a):=a$ and $\varphi^{n+1}(a):=\varphi\left(\varphi^{n}(a)\right)$ for $n \in \mathbb{N}_{0}:=\mathbb{N} \cup\{0\}$. It is then straightforward that the following defines an equivalence relation on $S$ :

(i) If $\varphi$ is a right shift, set $a \sim_{\varphi} a^{\prime}$ if and only if there is some $n \in \mathbb{N}_{0}$ such that $\varphi^{n}(a) \succeq a^{\prime}$ and $\varphi^{n}\left(a^{\prime}\right) \succeq a$ (equivalently for some $n, m \in \mathbb{N}_{0}, \varphi^{n}(a) \succeq$ $a^{\prime}$ and $\left.\varphi^{m}\left(a^{\prime}\right) \succeq a\right)$,

(ii) If $\varphi$ is a left shift, set $a \sim_{\varphi} a^{\prime}$ if and only if there is some $n \in \mathbb{N}_{0}$ such that $\varphi^{n}(a) \preceq a^{\prime}$ and $\varphi^{n}\left(a^{\prime}\right) \preceq a$ (equivalently for some $n, m \in \mathbb{N}_{0}, \varphi^{n}(a) \preceq$ $a^{\prime}$ and $\left.\varphi^{m}\left(a^{\prime}\right) \preceq a\right)$.

(iii) The equivalence classes $[a]_{\varphi}$ of $\sim_{\varphi}$ are $\preceq$-convex and closed under application of $\varphi$. By the $\preceq$-convexity, the quasi-order of $S$ induces an order on $S / \sim_{\varphi}$ such that $[a]_{\varphi} \prec[b]_{\varphi}$ if and only if $a^{\prime} \prec b^{\prime}$ for all $a^{\prime} \in[a]_{\varphi}$ and $b^{\prime} \in[b]_{\varphi}$.

Note that if $\varphi$ is the identity map $\mathbb{I}$, then the equivalence relation $\sim_{\mathbb{I}}$ is just $\asymp$ associated to the q.o., and is the finest one such that $S / \sim_{\mathbb{I}}$ is an ordered set.

We exploit Remark 3.1 to give an interpretation of the rank and principal rank as quotients via an appropriate equivalence relation, thereby providing - as promised in the previous section- alternative proofs for Theorem [2.7] and Theorem [2.12. It is precisely this approach that we will generalize to the difference rank in Section 5. Let $v$ be the natural valuation on the q.o. field $(K, \preceq)$. Recall that $\mathbf{P}_{K}$ denotes $K^{\succeq 0} \backslash K_{v}$. Consider the following commutative diagram:

$$
\begin{aligned}
& \mathbf{P}_{K} \stackrel{\varphi}{\longrightarrow} \mathbf{P}_{K} \\
& \downarrow v \mid v \\
& G^{<0} \stackrel{\varphi_{G}}{\longrightarrow} G^{<0} \quad \text { that is } \varphi_{G}(g)=2 g \text { for all } g \in G^{<0}, \varphi_{G} \text { is a left } \\
& \text { shift and } \\
& \text { with } \varphi(a):=a^{2} \text { for all } a \in \mathbf{P}_{K}, \varphi \text { is a right } \\
& \text { shift, } \\
& \varphi_{G}(v(a)):=v(\varphi(a)) \text { for all } a \in \mathbf{P}_{K}, \\
& v_{G}(G) \stackrel{\varphi_{\Gamma}}{\longrightarrow} v_{G}(G) \\
& \varphi_{\Gamma}\left(v_{G}(g)\right):=v_{G}\left(\varphi_{G}(g)\right) \text { for all } g \in G^{<0}, \\
& \text { that is } \varphi_{\Gamma}(\gamma)=\gamma \text { for all } \gamma \in \Gamma \text {, so that } \varphi_{\Gamma} \text { is } \\
& \text { just the identity map. }
\end{aligned}
$$

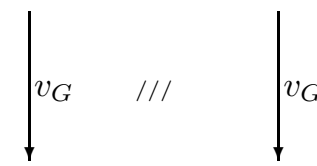

By Remark 3.1, we can work with the equivalence relations associated to the following oriented maps: the q.o. preserving map $\varphi$ and the order preserving maps $\varphi_{G}$ and $\varphi_{\Gamma}$ (as defined on the right hand side of the above diagram). Note that $\sim_{\varphi_{G}}$ is just archimedean equivalence on $G$ and $\sim_{\varphi_{\Gamma}}$ is just equality on $\Gamma$. The following straightforward observation will be useful for the proof of Theorem 3.4 below:

Lemma 3.2. The equivalence classes of $\sim_{\varphi}$ are closed under multiplication. 
Proof. The proof is similar to that of Lemma2.11, Let $a, b \in \mathbf{P}_{K}$, and without loss of generality assume that $a \preceq b$ and $a \sim_{\varphi} b$. We show that $a b \sim_{\varphi} a$. Let $n \in \mathbb{N}_{0}$, such that $b \preceq a^{2^{n}}$. By axiom qo2, $a b \preceq b^{2}$. Thus $b^{2} \preceq a^{2^{n}} b$ and $a b \preceq a^{2^{n}} b$. So, $a b \preceq a^{2^{n+1}}$. Since $1 \preceq b$, by axiom qo2, we get that $a \preceq a b$. Therefore, $a b \sim_{\varphi} a$.

Remark 3.3. We note that

$$
\varphi_{G}^{n}(v(a))=v\left(\varphi^{n}(a)\right) \text { and } \varphi_{\Gamma}^{n}\left(v_{G}(g)\right)=v_{G}\left(\varphi_{G}^{n}(g)\right)
$$

thus

(2) $a \sim_{\varphi} a^{\prime}$ if and only if $v(a) \sim_{\varphi_{G}} v\left(a^{\prime}\right)$ if and only if $v_{G}(v(a)) \sim_{\varphi_{\Gamma}} v_{G}\left(v\left(a^{\prime}\right)\right)$

Thus we have an order reversing bijection from $\mathbf{P}_{K} / \sim_{\varphi}$ onto $\Gamma / \sim_{\varphi_{\Gamma}}=\Gamma$. Thus the chain $\left[\mathbf{P}_{K} / \sim_{\varphi}\right]^{\text {is }}$ of non-empty initial segments of $\mathbf{P}_{K} / \sim_{\varphi}$ ordered by inclusion is isomorphic to $\Gamma^{\mathrm{fs}}$. In particular, initial segments which have a last element are in bijective correspondence to principal final segments. Thus the subchain of $\left[\mathbf{P}_{K} / \sim_{\varphi}\right]^{\text {is }}$ of initial segments which have a last element is isomorphic to $\Gamma^{*} 11$ Therefore, as promised in the previous section, Theorems 2.7 and 2.12 will now follow from the following result:

Theorem 3.4. The rank $\mathcal{R}$ is isomorphic to the chain $\left[\mathbf{P}_{K} / \sim_{\varphi}\right]^{\text {is }}$ and the principal rank $\mathcal{R}^{\text {pr }}$ is isomorphic to the subchain of $\left[\mathbf{P}_{K} / \sim_{\varphi}\right]{ }^{\text {is }}$ of initial segments which have a last element.

Proof. First we note that if $K_{w}$ is a convex valuation ring, then clearly $K_{w}^{\succ 0} \backslash K_{v}^{\succ 0}$ is an initial segment of $\mathbf{P}_{K}$. Moreover by Lemma 2.11 if $K_{w}$ is principal generated by $a$, then $[a]_{\sim_{\varphi}}$ is the last class. Furthermore, if $K_{w}$ intersects an equivalence class $[a]_{\sim_{\varphi}}$ then it must contain it, since the sequence $a^{n} ; n \in \mathbb{N}_{0}$ is cofinal in $[a]_{\sim_{\varphi}}$ and $K_{w}$ is a convex subring. We conclude that $\left(K_{w}^{\succ 0} \backslash K_{v}^{\succ 0}\right) / \sim_{\varphi}$ is an initial segment of $\mathbf{P}_{K} / \sim_{\varphi}$. Conversely set $\mathcal{I}_{w}=\left\{[a]_{\varphi} \mid a \in K_{w}^{\succ 0} \backslash K_{v}^{\succ 0}\right\}$. Given $\mathcal{I} \in\left[\mathbf{P}_{K} / \sim_{\varphi}\right]^{\text {is }}$, we show that there is a convex valuation ring $K_{w}$ such that $\mathcal{I}_{w}=\mathcal{I}$. Given $\mathcal{I}$, let $(\bigcup \mathcal{I})$ denote the set theoretic union of the elements of $\mathcal{I}$ and $-(\cup \mathcal{I})$ the set of additive inverses. Set $K_{w}=-(\bigcup \mathcal{I}) \cup K_{v} \cup(\cup \mathcal{I})$. We claim that $K_{w}$ is the required ring. Clearly, $\mathcal{I}_{w}=\mathcal{I}$. Further $K_{w}$ is convex (by its construction), and strictly contains $K_{v}$. We leave it to the reader, using Lemma 3.2 and Lemma 2.11, to verify that $K_{w}$ is a ring, and that $K_{w}$ is principal generated by $a$ if $[a]_{\sim \varphi}$ is the last element of $\mathcal{I}$.

\section{The Difference Analogue of the RAnK}

In this section, we develop a difference analogue of what has been reviewed above. That is, we develop a theory of difference compatible valuations, in analogy to the theory of convex valuations. The automorphism will play the role that multiplication plays in the previous case.

Let $(K, \preceq)$ be a q.o. field and $\sigma$ be a q.o. preserving field automorphism of $K$, that is, $a \preceq a^{\prime}$ if and only if $\sigma(a) \preceq \sigma\left(a^{\prime}\right)$, for all $a, a^{\prime} \in K$. We say that $(K, \preceq, \sigma)$ is a q.o. difference field.

\footnotetext{
${ }^{1}$ Note that the subchain of $\left[\mathbf{P}_{K} / \sim_{\varphi}\right]$ is of initial segments which have a last element is isomorphic to $\left[\mathbf{P}_{K} / \sim_{\varphi}\right]$ itself.
} 
Remark 4.1. Let $(K, \leq, \sigma)$ be an ordered difference field. Recall that the natural valuation $v$ on $K$ is defined by archimedean equivalence. Since archimedean equivalence is preserved under order preserving automorphisms, we see that $\sigma$ is also $\preceq_{v}$ preserving (so that $\left(K, \preceq_{v}, \sigma\right)$ is a q.o. difference field). The converse fails: Consider the field of real Laurent series $K:=\mathbb{R}((t))$ endowed with the lexicographic order and the corresponding natural valuation $v_{\min }$ (see definitions following Corollary 4.11 below). The map $t \mapsto(-t)$ defines a field automorphisme $\sigma$ on $K$ which clearly preserves $v_{\text {min }}$ but not the lexicographic order on $K$.

Now let $(K, \preceq, \sigma)$ be a non-trivial (i.e. $\sigma \neq$ identity) q.o. difference field and $v$ its natural valuation. By definition, $\sigma$ satisfies for all $a, b \in K: v(a) \leq$ $v(b)$ if and only if $v(\sigma(a)) \leq v(\sigma(b))$ and thus induces an order preserving automorphism $\sigma_{G}$ and $\sigma_{\Gamma}$ such that the following diagram commutes:

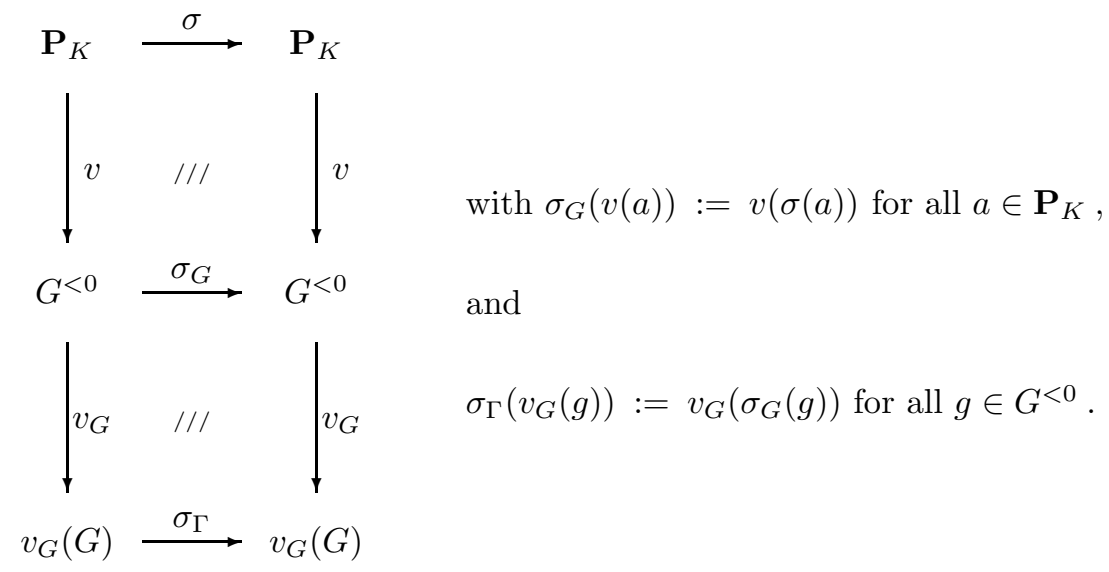

Now let $w$ be a convex valuation on $K$. Say $w$ is $\sigma$-compatible if for all $a, b \in K$ : $w(a) \leq w(b)$ if and only if $w(\sigma(a)) \leq w(\sigma(b))$. Thus $w$ is $\sigma$-compatible if and only if $\sigma$ preserves the q.o. $\preceq$.

The subset $\mathcal{R}_{\sigma}:=\left\{K_{w} \in \mathcal{R} ; w\right.$ is $\sigma$ - compatible $\}$ is the $\sigma$-rank of $(K, \preceq, \sigma)$. Similarly, the subset of all convex subgroups $G_{w} \neq\{0\}$ such that $\sigma_{G}\left(G_{w}\right)=G_{w}$, i.e $G_{w}$ is $\sigma_{G^{-}}$invariant, is the $\sigma$-rank of $G$. Finally, we denote by $\sigma_{\Gamma}-\Gamma^{\mathrm{fs}}$ the subset of non-empty final segments $\Gamma_{w}$ such that $\sigma_{\Gamma}\left(\Gamma_{w}\right)=\Gamma_{w}$, i.e. $\Gamma_{w}$ is $\sigma_{\Gamma^{-}}$ invariant.

The following Theorem 4.2, Lemmas 4.5 and 4.6 are analogues of Theorem 2.2 Lemma 2.5 and Lemma 2.6 respectively. They are verified by straightforward computations, using basic properties of valuations rings on the one hand and of automorphisms on the other (e.g. $\sigma(A \backslash B)=\sigma(A) \backslash \sigma(B), \sigma(A) \subseteq B$ if and only if $A \subseteq \sigma^{-1}(B)$ and $\sigma(A) \subseteq B$ if and only if $\sigma(-A) \subseteq-B$ ). The equivalence of 1) and 7) in Theorem 4.2 follows from the compatibility of $\sigma$ with $w$ on the one hand, and from the definition of the induced q.o. on $K w$ on the other. We call $K_{w}$ $\sigma$-compatible if any of the equivalent conditions below holds.

Theorem 4.2. The following assertions are equivalent for a convex valuation $w$ : 1) $w$ is $\sigma$-compatible 
2) $w$ is $\sigma^{-1}$-compatible

3) $\sigma\left(K_{w}\right)=K_{w}$

4) $\sigma\left(I_{w}\right)=I_{w}$

5) $\sigma\left(\mathcal{U}_{w}\right)=\mathcal{U}_{w}$

6) $\sigma\left(K_{w}^{\succ 0} \backslash K_{v}^{\succ 0}\right)=K_{w}^{\succ 0} \backslash K_{v}^{\succ 0}$

7) the map $\sigma w: K w \rightarrow K w$ defined by aw $\mapsto \sigma(a) w$ is well-defined and is a q.o. (with respect to the induced q.o. on $K w$ ) preserving field automorphism of $K w$.

Remark 4.3. Let $(K, \leq, \sigma)$ be an ordered field with natural valuation $v$. In this case, condition 7) on $\sigma w$ in Theorem 4.2 is referring to the induced order on the residue field $K w$. Consider instead the following condition:

8) the map $\sigma w: K w \rightarrow K w$ defined by $a w \mapsto \sigma(a) w$ is well-defined and is a q.o. (with respect to the q.o. $\preceq_{v / w}$ on $K w$ ) preserving field automorphism of $K w$.

We observe that 7) implies 8). Indeed, $\sigma w$ is assumed to be order preserving on $K w$ by 7 ). Now $(K w)(v / w)=K v$ (see [17, Lemma 2.1]). Therefore $v / w$ has archimedean residue field and is thus the natural valuation on the ordered field $K w$. By Remark 4.1 we obtain the assertion.

Remark 4.4. The maximal ideals $I_{w}$ appearing in Theorem 4.24$)$ are $\sigma$-invariant prime ideals (also called transformally prime ideals in [4) of the valuation ring $K_{v}$ and the coarsenings $K_{w}$ are just the localizations of $K_{v}$ at those $\sigma$-invariant prime ideals, see [5, Lemma 2.3.1 p. 43]. Thus the $\sigma$-rank is also characterized by the chain of $\sigma$ - invariant prime ideals of $K_{v}$.

Lemma 4.5. The correspondence $K_{w} \mapsto G_{w}$ is an order preserving bijection from $\mathcal{R}_{\sigma}$ onto the $\sigma_{G}$-rank of $G$.

Lemma 4.6. The correspondence $G_{w} \mapsto \Gamma_{w}$ is an order preserving bijection from the $\sigma_{G}$-rank of $G$ onto $\sigma_{\Gamma}-\Gamma^{\mathrm{fs}}$.

We deduce from Lemma 4.5 and Lemma 4.6 that the $\sigma$-rank is the order type of $\sigma_{\Gamma}-\Gamma^{\mathrm{fs}}$ :

Theorem 4.7. The correspondence $K_{w} \mapsto \Gamma_{w}$ is an order preserving bijection from $\mathcal{R}_{\sigma}$ onto $\sigma_{\Gamma}-\Gamma^{\mathrm{fs}}$.

We now exploit this observation. An automorphism $\sigma$ is an isometry if $v(\sigma(a))=$ $v(a)$ for all $a \in K$, equivalently $\sigma_{G}$ is the identity automorphism, and a weak isometry if $\sigma_{\Gamma}$ is the identity automorphism. Every isometry is a weak isometry. Note that if $\Gamma$ is a rigid chain (i.e the only order preserving automorphism is the identity map), then $\sigma$ is necessarily a weak isometry. If $\sigma$ is a weak isometry, then $\sigma_{\Gamma}\left(v_{G}(g)\right)=v_{G}\left(\sigma_{G}(g)\right)=v_{G}(g)$, thus $g$ is archimedean equivalent to $\sigma_{G}(g)$ for all $g$, and so every convex subgroup is $\sigma_{G}$-invariant.

Corollary 4.8. If $\sigma$ is a weak isometry, then $\mathcal{R}_{\sigma}=\mathcal{R}$.

Corollary 4.9. The correspondence $K_{w} \mapsto \min \Gamma_{w}$ is an order (reversing) isomorphism from $\mathcal{R}_{\sigma} \cap \mathcal{R}^{\mathrm{pr}}$ onto the chain $\left\{\gamma ; \sigma_{\Gamma}(\gamma)=\gamma\right\}$ of fixed points of $\sigma_{\Gamma}$.

Proof. By Lemma 2.9 set $\min \Gamma_{w}:=\gamma_{0}$. By Lemma 4.5 and Lemma 4.6, $\Gamma_{w}$ in invariant under $\sigma_{\Gamma}$. Since $\sigma_{\Gamma}$ is order preserving, we must have $\sigma_{\Gamma}\left(\gamma_{0}\right)=\gamma_{0}$

At the other extreme $\sigma$ is said to be $\omega$-increasing if $a^{n} \prec \sigma(a)$ for all $n \in \mathbb{N}_{0}$ and all $a \in \mathbf{P}_{K}$, and $\omega$-contracting if $\sigma^{-1}$ is $\omega$-increasing. 
Remark 4.10. Note that $\sigma$ is $\omega$-increasing (respectively, $\omega$-contracting) if and only if $\sigma_{\Gamma}$ is a strict left shift, that is, $\sigma_{\Gamma}(\gamma)<\gamma$ for all $\gamma \in \Gamma$ (respectively, a strict right shift, i.e. $\sigma_{\Gamma}(\gamma)>\gamma$ for all $\left.\gamma \in \Gamma\right)$. Thus if $\sigma \omega$-increasing or $\omega$-contracting, then $\sigma_{\Gamma}$ has no fixed points.

Corollary 4.11. If $\sigma$ is $\omega$-increasing or $\omega$-contracting, then $\mathcal{R}_{\sigma} \cap \mathcal{R}^{\mathrm{pr}}$ is empty.

Recall that the Hahn group 9] over the chain $\Gamma$ and components $\mathbb{R}$, denoted $\mathbf{H}_{\Gamma} \mathbb{R}$, is the totally ordered abelian group whose elements are formal sums $g:=\sum g_{\gamma} 1_{\gamma}$, with well-ordered support $g:=\left\{\gamma ; g_{\gamma} \neq 0\right\}$. Here $g_{\gamma} \in \mathbb{R}$ and $1_{\gamma}$ denotes the characteristic function on the singleton $\{\gamma\}$. Addition is pointwise and the order lexicographic. Similarly, given a field $F$, the field of generalized power series over the ordered abelian group $G$ (or Hahn field over $G$ ) with coefficients in $F$, denoted $\mathbb{F}:=F((G))$, is the field whose elements are formal series $s:=\sum s_{g} t^{g}$, with well-ordered support $s:=\left\{g ; s_{g} \neq 0\right\}$. Addition is pointwise, multiplication is given by the usual convolution formula. The field $\mathbb{F}$ has the same characteristic as that of $F$. The canonical valuation $v_{\min }$ on $\mathbb{F}$ is defined by $v_{\min }(s):=\min$ support $s$ for $s \neq 0$. Its value group is $G$ and its residue field is $F$. Thus ( $\left.\mathbb{F}, \preceq_{v_{\min }}\right)$ is a q.o. field. If $F$ is an ordered field, its order extends to the lexicographic order on $\mathbb{F}$ : a series $s$ is positive if and only if the coefficient of $t^{v_{\min }(s)}$ is positive in $F$. Thus, in that case $(\mathbb{F}, \leq)$ is an ordered field. Hahn fields are maximally valued: they admit no proper immediate extension, that is, no proper valued field extension preserving the value group and the residue field. They were extensively studied e.g. by Hahn [9] and in the seminal paper of Kaplansky [13].

Lemma 4.12. Any order preserving automorphism $\sigma_{\Gamma}$ of the chain $\Gamma$ lifts to an order preserving automorphism $\sigma_{G}$ of the Hahn group $G$ over $\Gamma$, and $\sigma_{G}$ lifts in turn to a q.o. preserving automorphism $\sigma$ of the Hahn field over $G$.

Proof. Set $\sigma_{G}\left(\sum g_{\gamma} 1_{\gamma}\right):=\sum g_{\gamma} 1_{\sigma_{\Gamma}(\gamma)}$. It is straightforward to verify that the thus defined $\sigma_{G}$ induces the given automorphism $\sigma_{\Gamma}$ on $\Gamma$. Thus $\sigma_{G}$ is a lifting of $\sigma_{\Gamma}$. Now set $\sigma\left(\sum s_{g} t^{g}\right):=\sum s_{g} t^{\sigma_{G}(g)}$. Again, it is clear that $\sigma$ induces $\sigma_{G}$ on $G$. Thus $\sigma$ is a lifting of $\sigma_{G}$ as asserted.

Corollary 4.13. Given any order type $\tau$ there exists an ordered difference field $(K, \leq, \sigma)$, and also a p.q.o. difference field $(K, \preceq, \sigma)$ such that the order type of $\mathcal{R}_{\sigma} \cap \mathcal{R}^{\mathrm{pr}}$ is $\tau$.

Proof. Set $\mu:=\tau^{*}$, and consider e.g. the linear ordering $\Gamma:=\sum_{\mu} \mathbb{Q}^{\geq 0}$, that is, the concatenation of $\mu$ copies of the non-negative rationals. Fix a non-trivial order automorphism $\eta$ of $\mathbb{Q}^{>0}$. Define $\sigma_{\Gamma}$ to be the uniquely defined order automorphism of $\Gamma$ fixing every $0 \in \mathbb{Q}^{\geq 0}$ in every copy and extending $\eta$ on every copy. It is clear that the order type of the chain of fixed points (the zeros in every copy) of $\sigma_{\Gamma}$ is $\mu$. Set e.g. $G:=\mathbf{H}_{\Gamma} \mathbb{R}$. By Lemma 4.12, $\sigma_{\Gamma}$ lifts canonically to $\sigma_{G}$ on $G$. Now consider e.g. the ordered field $\mathbb{F}:=\mathbb{R}((G))$. Again by Lemma 4.12, $\sigma_{G}$ lifts canonically to an order automorphism $\sigma$ of $\mathbb{F}$. This is our required $\sigma$, by Corollary 4.9. To obtain a p.q.o difference field, take $F$ any field and the corresponding $\left(\mathbb{F}, \preceq_{v_{\min }}, \sigma\right)$.

In the next section, we will exploit appropriate equivalence relations to define the principal difference rank and construct difference fields of arbitrary principal difference rank. 


\section{ThE $\sigma$-RANK AND PRINCIPAL $\sigma$-RANK VIA EQUIVALENCE RELATIONS}

Let $(K, \preceq, \sigma)$ be a q.o. difference field. As promised in Section 3, we now exploit Remark 3.1 to give an interpretation of the $\sigma$ - rank and define the principal $\sigma$-rank as quotients via appropriate equivalence relations. Our aim is to state and prove the analogues to Theorems [3.4, 2.7 and 2.12, We recall that the q.o. preserving maps considered in Remark 3.1 are assumed to be oriented. Moreover, scrutinizing the proof of Theorem 3.4 we quickly realize that we need Lemma 5.2 below, an analogue of Lemma 3.2. Thus we need further assumptions on $\sigma$, to ensure that $\sigma$ satisfies Lemma 5.2. For simplicity from now on we will assume that $\sigma$ or $\sigma^{-1}$ satisfy $\sigma(a) \succeq a^{2}$ for all $a \in \mathbf{P}_{K}$. Note that this implies that $\sigma(a) \succ a$, so $\sigma$ is an oriented strict right-shift. Note that our condition on $\sigma$ is fulfilled for $\omega$-increasing or $\omega$-contracting automorphisms.

A convex subring $K_{w} \neq K_{v}$ is $\sigma$-principal generated by $a$ for $a \in \mathbf{P}_{K}$ if $K_{w}$ is the smallest convex $\sigma$-compatible subring containing $a$. The $\sigma$-principal rank of $K$ is the subset $\mathcal{R}_{\sigma}^{\mathrm{pr}}$ of $\mathcal{R}_{\sigma}$ consisting of all $\sigma$-principal $K_{w} \in \mathcal{R}$. We will use the analogue of Remark 3.3

Remark 5.1. The maps $\sigma, \sigma_{G}$ and $\sigma_{\Gamma}$ are q.o. preserving and we can define the corresponding equivalence relations $\sim_{\sigma}, \sim_{\sigma_{G}}$ and $\sim_{\sigma_{\Gamma}}$. As before we have

$$
a \sim_{\sigma} a^{\prime} \text { if and only if } v(a) \sim_{\sigma_{G}} v\left(a^{\prime}\right) \text { if and only if } v_{G}(v(a)) \sim_{\sigma_{\Gamma}} v_{G}\left(v\left(a^{\prime}\right)\right)
$$

Thus we have an order reversing bijection from $\mathbf{P}_{K} / \sim_{\sigma}$ onto $\Gamma / \sim_{\sigma_{\Gamma}}$. Thus the chain $\left[\mathbf{P}_{K} / \sim_{\sigma}\right]^{\text {is }}$ of initial segments of $\mathbf{P}_{K} / \sim_{\sigma}$ ordered by inclusion is isomorphic to $\left(\Gamma / \sim_{\sigma_{\Gamma}}\right)^{\text {fs }}$. As before, the subchain of initial segments which have a last element is isomorphic to $\left(\Gamma / \sim_{\sigma_{\Gamma}}\right)^{*}$.

Lemma 5.2. The equivalence classes of $\sim_{\sigma}$ are closed under $\sigma$ and under multiplication.

Proof. The condition on $\sigma$ implies by induction that $\sigma^{n}(a) \succeq a^{2^{n}}$. Thus given $n \in \mathbb{N}_{0}$, there exists $l \in \mathbb{N}_{0}$ such that $\sigma^{l}(a) \succeq a^{n}$. Thus $a \sim_{\sigma} \sigma(a)$. So the equivalence classes of $\sigma$ are closed under $\sigma$. Recall that the natural valuation $v_{G}$ on $G$ satisfies $v_{G}(x+y)=\min \left\{v_{G}(x), v_{G}(y)\right\}$ if $\operatorname{sign}(x)=\operatorname{sign}(y)$. Again one easily deduces from this fact and the equivalences (3) above that the equivalence classes of $\sigma$ are closed under multiplication. Indeed assume that $a \sim_{\sigma} b$ and $a \sim_{\sigma} c$. We want to show that $a \sim_{\sigma} b c$. Set $x:=v(b), y:=v(c)$ and $z:=v(a) \in G^{<0}$. By the first equivalence in (3), it is enough to show that $v(a) \sim_{\sigma_{G}} v(b c)$ i.e. that $x+y \sim_{\sigma_{G}} z$. By the second equivalence in (3), it is enough to show that $v_{G}(x+y) \sim_{\sigma_{\Gamma}} v_{G}(z)$. Without loss of generality $v_{G}(x+y)=v_{G}(x)$. But since $a \sim_{\sigma} b$ it follows by (3) that $v_{G}(x) \sim_{\sigma_{\Gamma}} v_{G}(z)$ as required.

We can now prove the analogue of Theorem 3.4

Theorem 5.3. The $\sigma$-rank $\mathcal{R}_{\sigma}$ is isomorphic to $\left[\mathbf{P}_{K} / \sim_{\sigma}\right]^{\text {is }}$ and the principal $\sigma$ rank $\mathcal{R}_{\sigma}^{\mathrm{pr}}$ is isomorphic to the subset of $\left[\mathbf{P}_{K} / \sim_{\sigma}\right]^{\text {is }}$ of initial segments which have a last element 2

\footnotetext{
${ }^{2}$ Note that the subchain of $\left[\mathbf{P}_{K} / \sim_{\sigma}\right]^{\text {is }}$ of initial segments which have a last element is isomorphic to $\left[\mathbf{P}_{K} / \sim_{\sigma}\right]$ itself.
} 
Proof. First we note that if $K_{w}$ is a convex $\sigma$-compatible valuation ring, then clearly $K_{w}^{\succ 0} \backslash K_{v}^{\succ 0}$ is an initial segment of $\mathbf{P}_{K}$. Furthermore, if $K_{w}$ intersects a $\sigma$ equivalence class $[a]_{\sim_{\sigma}}$ then it must contain it, since the sequence $\sigma(a)^{n} ; n \in \mathbb{N}_{0}$ is cofinal in $[a]_{\sim_{\sigma}}$ and $K_{w}$ is a convex subring. We conclude that $\left(K_{w}^{\succ 0} \backslash K_{v}^{\succ 0}\right) / \sim_{\sigma}$ is an initial segment of $\mathbf{P}_{K} / \sim_{\sigma}$ and moreover $[a]_{\sim_{\sigma}}$ is the last class in case $K_{w}$ is $\sigma$ - principal generated by $a$. Conversely set $\mathcal{I}_{w}=\left\{[a]_{\sigma} \mid a \in K_{w}^{\succ 0} \backslash K_{v}^{\succ 0}\right\}$. Given $\left.\mathcal{I} \in\left[\mathbf{P}_{K} / \sim_{\sigma}\right]\right]^{\text {is }}$, we show that there is a $\sigma$-compatible convex valuation ring $K_{w}$ such that $\mathcal{I}_{w}=\mathcal{I}$. Given $\mathcal{I}$, let $(\bigcup \mathcal{I})$ denote the set theoretic union of the elements of $\mathcal{I}$ and $-(\bigcup \mathcal{I})$ the set of additive inverses. Set $K_{w}=-(\bigcup \mathcal{I}) \cup K_{v} \cup(\bigcup \mathcal{I})$. We claim that $K_{w}$ is the required ring. Clearly, $\mathcal{I}_{w}=\mathcal{I}$. Further $K_{w}$ is convex (by its construction), and strictly contains $K_{v}$. We leave it to the reader, using Lemma 5.2 to verify that $K_{w}$ is a $\sigma$-compatible subring, and that $K_{w}$ is $\sigma$-principal generated by $a$ if $[a]_{\sim \sigma}$ is the last element of $\mathcal{I}$.

We now deduce from this theorem combined with Remark 5.1 the promised analogues of Theorems 2.7 and 2.12 respectively:

Corollary 5.4. $\mathcal{R}_{\sigma}$ is (isomorphic to) $\left(\Gamma / \sim_{\sigma_{\Gamma}}\right)^{\mathrm{fs}}$.

Corollary 5.5. $\mathcal{R}_{\sigma}^{\mathrm{pr}}$ is (isomorphic to) $\left(\Gamma / \sim_{\sigma_{\Gamma}}\right)^{*}$.

We call the order type of $\left(\Gamma / \sim_{\sigma_{\Gamma}}\right)$ the rank of the automorphism $\sigma_{\Gamma}$. We now can construct $\omega$-increasing automorphisms of arbitrary principal difference rank. Corollary [5.6 below, compared to Corollary 4.11 demonstrates the discrepancy between the chains $\mathcal{R}_{\sigma}^{\mathrm{pr}}$ and $\mathcal{R}_{\sigma} \cap \mathcal{R}^{\mathrm{pr}}$.

Corollary 5.6. Given any order type $\tau$ there exists a maximally valued ordered field endowed with an $\omega$-increasing automorphism of principal difference rank $\tau$.

Proof. Set $\mu:=\tau^{*}$, and consider e.g. the linear ordering $\Gamma:=\sum_{\mu} \mathbb{Q}$, that is, the concatenation of $\mu$ copies of the non-negative rationals. Let $\ell$ be e.g. translation by -1 on $\mathbb{Q}$. Define $\sigma_{\Gamma}$ to be the uniquely defined order automorphism of $\Gamma$ extending $\ell$ on every copy. It is clearly a strict left shift of rank $\mu$. Set e.g. $G:=\mathbf{H}_{\Gamma} \mathbb{R}$. Then by Lemma $4.12 \sigma_{\Gamma}$ lifts canonically to $\sigma_{G}$ on $G$. Now set e.g. $K:=\mathbb{R}((G))$. By Lemma 4.12, Remark 4.10 and Corollary 5.5, $\sigma_{G}$ lifts canonically to an $\omega$-increasing automorphism of $K$ of principal difference rank $\mu^{*}=\tau$.

Example 5.7. Consider the chain $\Gamma=\mathbb{Z} \times \mathbb{Z}$ (the lexicographic product of two copies of $\mathbb{Z})$. We endow $\Gamma$ with the automorphisms $\tau((x, y)):=(x-1, y)$ and $\sigma((x, y)):=(x, y-1)$. The rank of $\tau$ is one and that of $\sigma$ is $\mathbb{Z}$. Both are strict left shifts. Lifting those automorphisms to $G:=\mathbf{H}_{\Gamma} \mathbb{R}$ and then to $K:=\mathbb{R}((G))$ as in the proof of Corollary [5.6, we obtain $\omega$-increasing automorphisms of $K$ of distinct principal difference ranks.

For a regular uncountable cardinal $\kappa$, let us denote by $G_{\kappa}$ the $\kappa$-bounded Hahn group, that is, the subgroup of $G=\mathbf{H}_{\Gamma} \mathbb{R}$ consisting of elements with support of cardinality $<\kappa$. Similarly, we denote by $\mathbb{R}((G))_{\kappa}$ the $\kappa$-bounded Hahn field, i.e. the subfield of $K=\mathbb{R}((G))$ consisting of series with support of cardinality $<\kappa$. If $\kappa=\kappa^{<\kappa}$ then $\mathbb{R}\left(\left(G_{\kappa}\right)\right)_{\kappa}$ has cardinality $\kappa$, see 1$]$.

We now generalize Example 5.7. In [18, Corollary 14], we construct for every infinite cardinal $\kappa$ a chain $\Gamma$ of cardinality $\kappa$ which admits of family of $2^{\kappa}$ strict left shift automorphisms, of pairwise distinct ranks. Lifting those automorphisms to $\mathbb{R}\left(\left(G_{\kappa}\right)\right)_{\kappa}$, we conclude as in [18, Theorem 9]: 
Theorem 5.8. Let $\kappa=\kappa^{<\kappa}$ be a regular uncountable cardinal and $\Gamma$ be any chain of cardinality $\kappa$ which admits a family of $2^{\kappa}$ strict left shift automorphisms of pairwise distinct ranks. Then the corresponding $\kappa$-bounded Hahn field $\mathbb{R}\left(\left(G_{\kappa}\right)\right)_{\kappa}$ of cardinality $\kappa$ admits a family of $2^{\kappa} \omega$-increasing automorphisms of distinct principal difference ranks.

\section{REFERENCES}

[1] Alling, N.L. and Kuhlmann, S. : On $\eta_{\alpha}$-groups and fields, Order 11 (1994), 85-92.

[2] Birkhoff, G. : Lattice theory, American Math. Soc. (1948).

[3] Brown, R. : Automorphisms and isomorphisms of real Henselian fields, Trans. Amer. Math. Soc. 307 (1988), 675-703.

[4] Cohn, R.: Difference algebra, Interscience Publishers John Wiley and Sons, New-YorkLondon-Sydney (1965).

[5] Engler, A. J. and Prestel, A.: Valued Fields, Springer Monographs in Math. (2005).

[6] Fakhruddin, S.M.: Quasi-ordered fields, J. Pure Appl. Algebra 45 (1987), 207-210.

[7] Fuchs, L.: Partially ordered algebraic systems, Pergamon Press (1963).

[8] Droste, M. and Göbel, R.: The automorphism groups of Hahn groups, Ordered algebraic structures (Curacao, 1995), Kluwer Acad. Publ.,Dordrecht (1997), 183-215.

[9] Hahn, H. : Über die nichtarchimedischen Grössensystem, Sitzungsberichte der Kaiserlichen Akademie der Wissenschaften, Mathematisch - Naturwissenschaftliche Klasse (Wien) 116 (1907), no. Abteilung IIa, 601-655.

[10] Hardy, G. H. : Orders of Infinity; The "Infinitärcalcul" of Paul Du Bois-Reymond, Cambridge University Press (1910).

[11] Hofberger, H.: Automorphismen formal reeller Körper, $\mathrm{PhD}$ thesis Ludwig-MaximiliansUniversität München (1991).

[12] Haskell, D. and Macpherson, D. : Cell decompositions of C-minimal structures, Annals of Pure and Applied Logic 66 (1994) 113-162.

[13] Kaplansky, I.: Maximal fields with valuations I, Duke Math. J. 9 (1942), 303-321.

[14] Kuhlmann, S. : Ordered exponential fields, Fields Institute Monographs 12 (2000).

[15] Krivine, J. L. : Anneaux préordonnés, Journal d'analyse mathématique 12 (1964), 307-326.

[16] Kruskal, J. B. : The Theory of Well-Quasi-Ordering: A Frequently Discovered Concept, Journal of Combinatorial Theory (A) 13 (1972), 297-305.

[17] Kuhlmann, F.-V., Kuhlmann, S.:Valuation theory of exponential Hardy fields II: Principal parts of germs in the Hardy field of o-minimal exponential expansions of the reals, to appear in AMS Contemporary Mathematics (CONM); Proceedings of the Conference on Ordered Algebraic Structures and Related Topics. Edited by: F. Broglia, F. Delon, M. Dickmann, D. Gondard, and V. Powers (2016).

[18] Kuhlmann, S., Shelah, S. : $\kappa$ - bounded Exponential-Logarithmic Power Series Fields, Annals Pure and Applied Logic 136, (2005), 284-296.

[19] Lam, T. Y.: The theory of ordered fields, in: Ring Theory and Algebra III (ed. B. McDonald), Lecture Notes in Pure and Applied Math. 55 Dekker, New York (1980), 1-152.

[20] Lam, T. Y.: Orderings, valuations and quadratic forms, Amer. Math. Soc. Regional Conference Series in Math. 52, Providence (1983).

[21] Lang, S.: The theory of real places, Ann. Math. 57 (1953), 378-391.

[22] Lehéricy, G. : The differential rank of a valued differential field, Dissertation, in preparation (2016).

[23] Prieß-Crampe, S.: Angeordnete Strukturen. Gruppen, Körper, projektive Ebenen, Ergebnisse der Mathematik und ihrer Grenzgebiete 98, Springer (1983).

[24] Prestel, A.: Lectures on Formally Real Fields, Springer Lecture Notes in Math. 1093 (1984).

[25] Rosenstein J. G. : Linear orderings, Pure and Applied Mathematics 98, Academic Press, Inc., New York-London (1982).

[26] Schilling O.F.G.: Automorphisms of fields of formal power series, Bull. Amer. Math. Soc. 50, (1944), 892-901.

[27] Zariski, O. and Samuel, P.: Commutative algebra, Vol. II. The University Series in Higher Mathematics. D. Van Nostrand Co., Inc., Princeton, N. J.-Toronto-London-New York (1960). 
THE VALUATION DIFFERENCE RANK OF A QUASI-ORDERED DIFFERENCE FIELD 15

Universität Konstanz, FB Mathematik und Statistik, 78457 Konstanz, Germany

E-mail address: salma.kuhlmann@uni-konstanz.de

imB, Université Bordeaux 1, 33405 Talence, France

E-mail address: mmatusin@math.u-bordeaux1.fr

Institut de mathématique, Le Pentagone, Université de Mons, B-7000 Mons, Belgium

E-mail address: Francoise.Point@umons.ac.be 